\title{
Hydrothermal Synthesis of Single-Crystalline Antimony Telluride Nanobelts
}

\author{
Supporting Information
}

Weidong Shi, Jiangbo Yu, Haishui Wang and Hongjie Zhang*

Key Laboratory of Rare Earth Chemistry and Physics, Changchun Institute of Applied Chemistry, Chinese Academy of Sciences, Changchun, 130022, P. R. China.

Correspondence Author: Hongjie Zhang (hongjie@ns.ciac.jl.cn)

Supporting Information 1 The detailed scheme of the synthetic process of $\mathrm{Sb}_{2} \mathrm{Te}_{3}$ colloid.

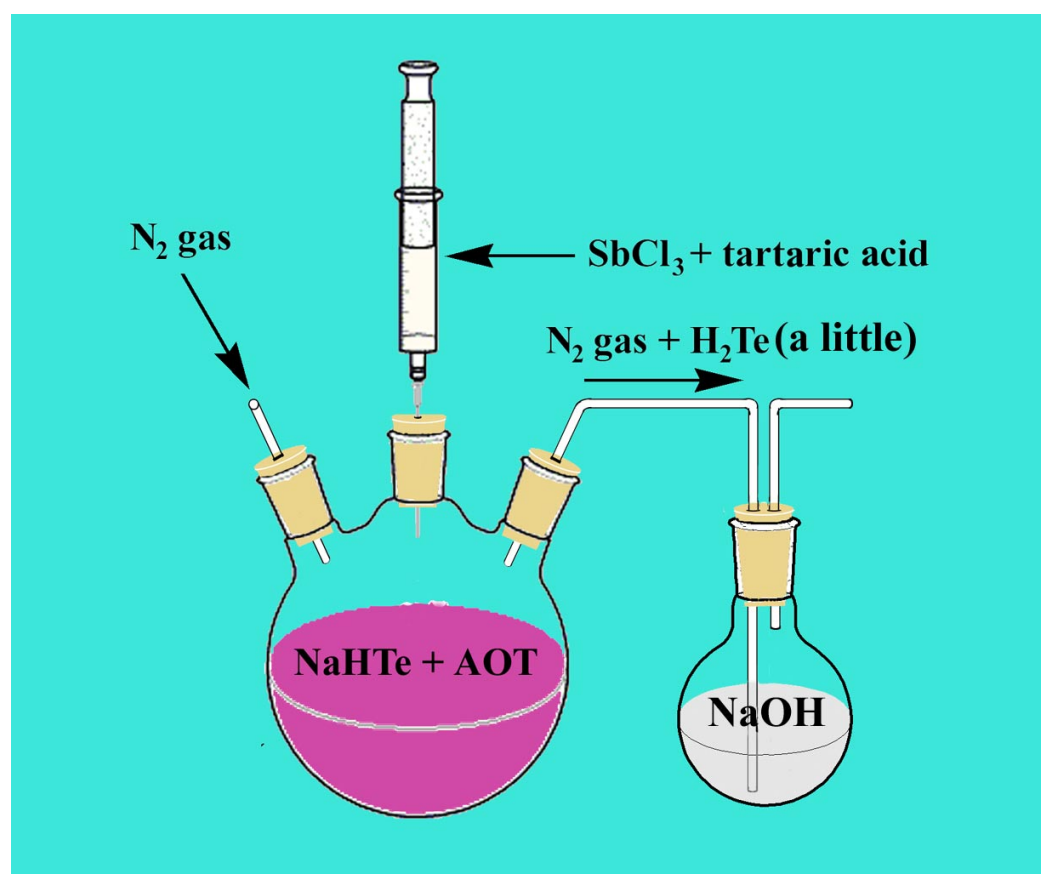


Supporting Information 2 Elemental analysis of the resulting nanobelts using secondary electrons by SEM.

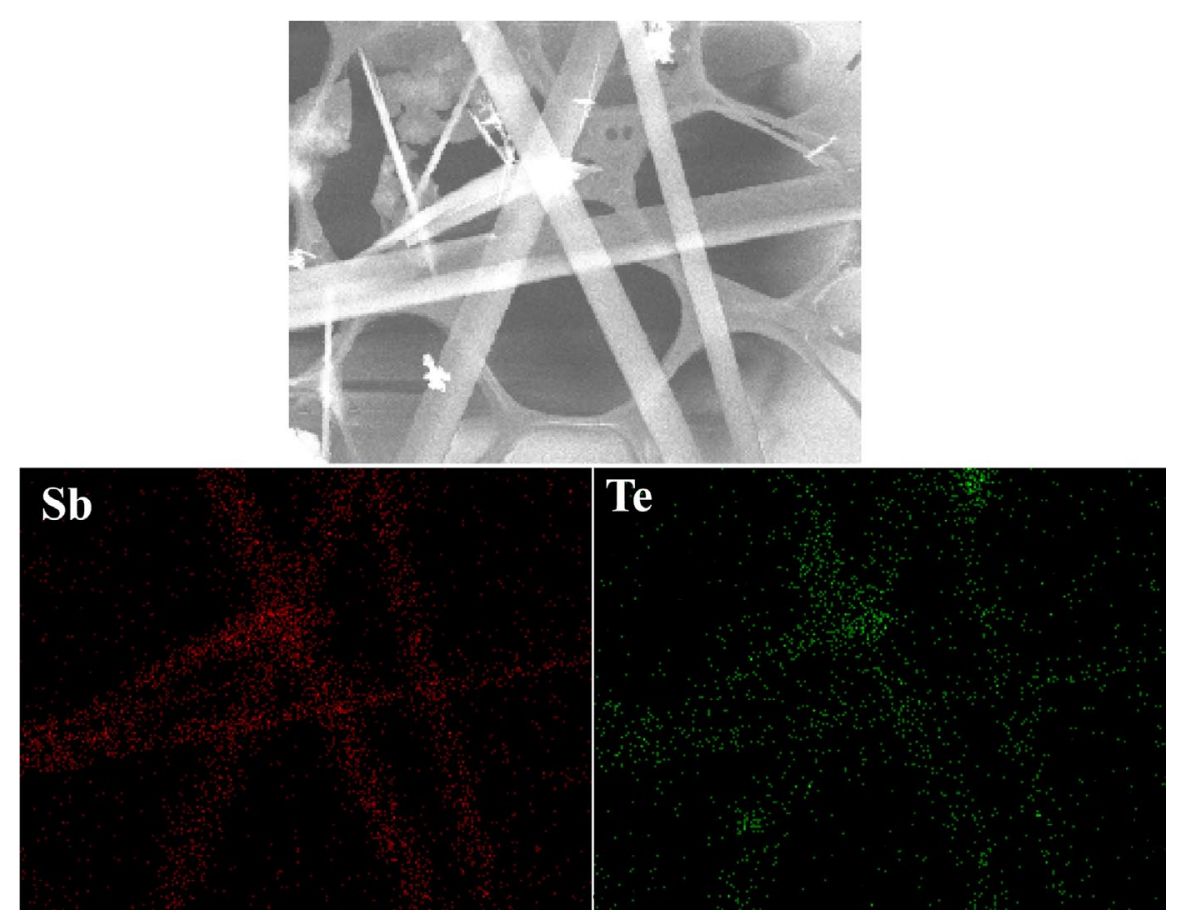


Supporting Information $3 \mathrm{SEM}$ images of the obtained $\mathrm{Sb}_{2} \mathrm{Te}_{3}$ products without adding AOT.
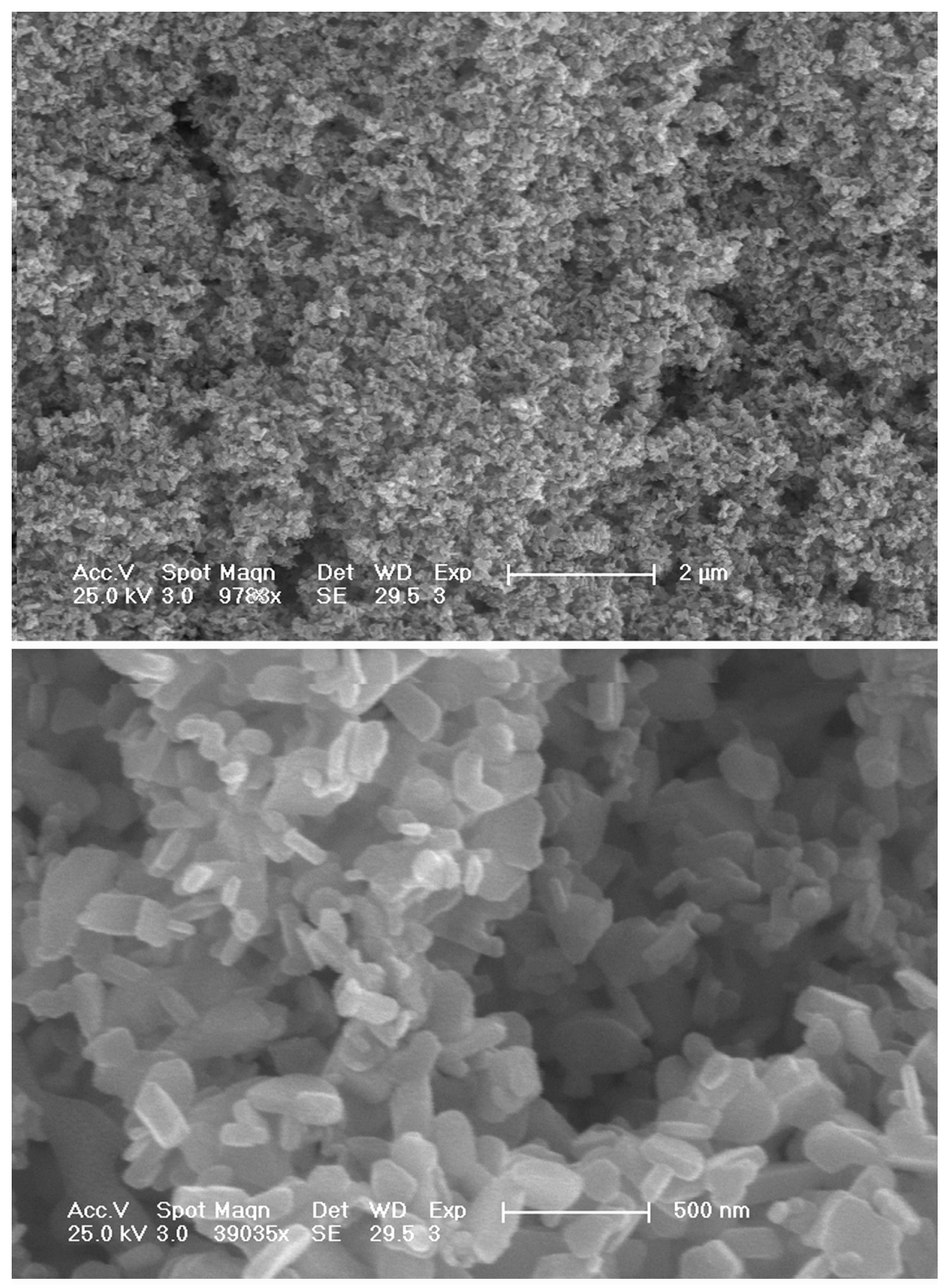
Supporting Information $4 \mathrm{SEM}$ images of the obtained $\mathrm{Sb}_{2} \mathrm{Te}_{3}$ products with adding $0.2 \mathrm{~g}$ AOT.
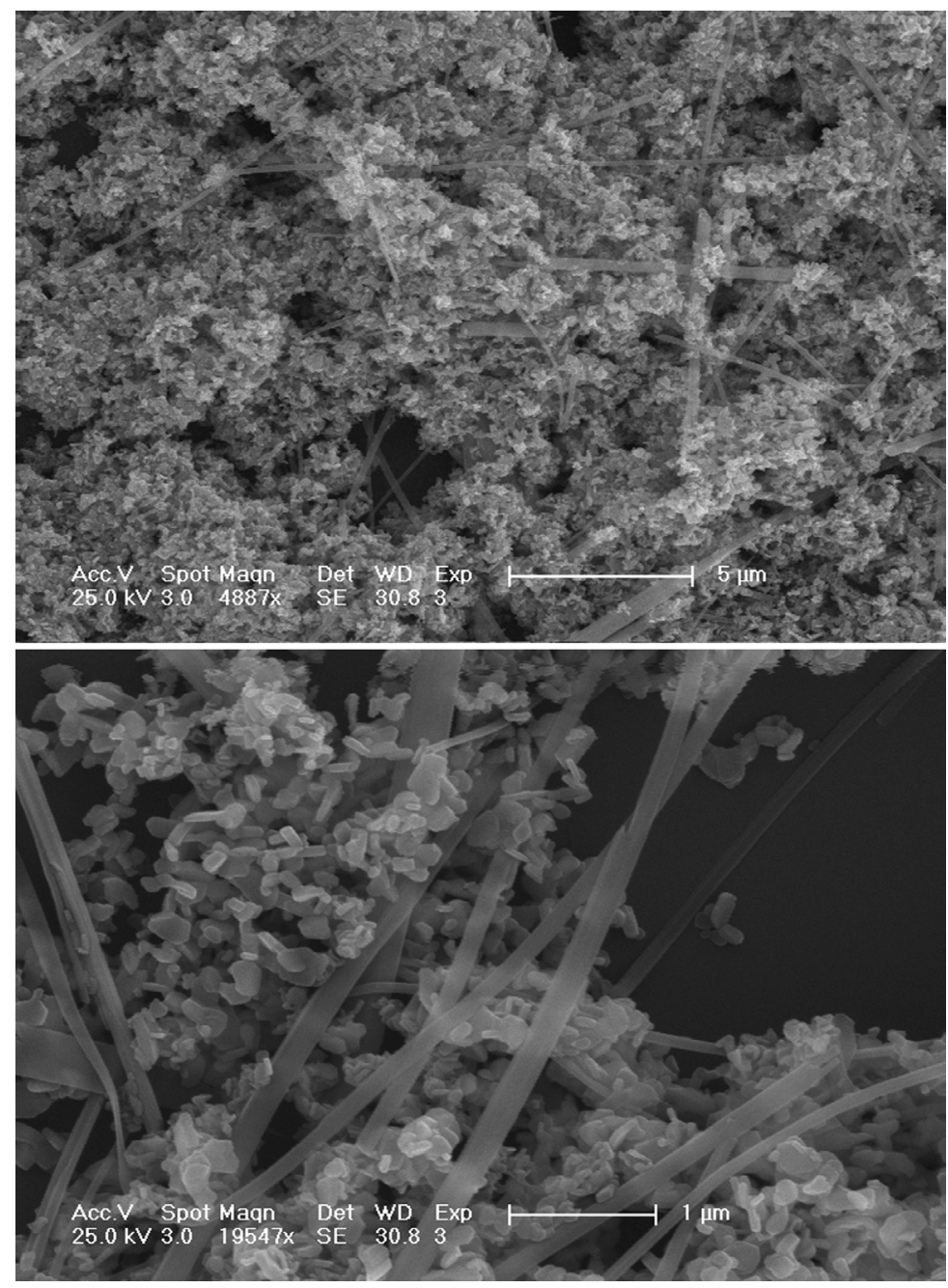